Canadian Oncology

Nursing Journal

Revue canadienne

de soins infirmiers

en oncologie

Volume 31, Issue 3 • Summer 2021

elSSN: 2368-8076 


\title{
Cancer du sein chez les Rwandaises en âge de procréer du district sud de Kayonza : facteurs influençant les pratiques de dépistage
}

\author{
par Pierre Céléstin Igiraneza, Lilian A. Omondi, Bellancille Nikuze, Marie Goretti Uwayezu, Margaret Fitch, Gaudence Niyonsenga
}

\section{RÉSUMÉ}

Introduction : Au Rwanda, le cancer du sein est une grande préoccupation pour la santé des femmes puisque la plupart des diagnostics sont faits à un stade avancé de la maladie. On sait pourtant que les pratiques de dépistage du cancer du sein permettent d'accélérer le diagnostic.

Objectif: Évaluer les facteurs qui influencent les pratiques de dépistage du cancer du sein chez les femmes en âge de procréer habitant le district de Kayonza, au Rwanda.

Méthodologie : Une étude quantitative a été menée dans quatre centres de santé au sud du district de Kayonza auprès de 246 fermmes de 16 à 49 ans. Un questionnaire structuré, prétesté et autoadministré a été utilisé pour évaluer l'influence des connaissances, des centres de santé et des facteurs socioéconomiques et culturels sur les pratiques de dépistage du cancer du sein chez les femmes dans le sud de Kayonza.

\section{AUTEURS

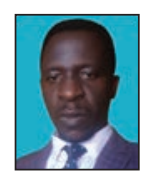 \\ Pierre Céléstin Igiraneza*, Camp de réfugiés Alight Rwanda de Nyabiheke, Rwanda}

Lilian A. Omondi, École de sciences infirmières et pratique sagefemme, Université du Rwanda, Collège de médecine et de sciences de la santé, Kigali, Rwanda, Collège des sciences infirmières Rory Meyers de l'Université de New York et École des sciences infirmières de l'Université de Nairobi, Kenya

Bellancille Nikuze, École de sciences infirmières et pratique sagefemme, Université du Rwanda, Collège de médecine et de sciences de la santé, Kigali, Rwanda, Collège des sciences infirmières Rory Meyers de l'Université de New York et École des sciences infirmières de l'Université de Nairobi, Kenya

Marie Goretti Uwayezu, École de sciences infirmières et pratique sage-femme, Université du Rwanda, Collège de médecine et de sciences de la santé, Kigali, Rwanda, Collège des sciences infirmières Rory Meyers de l'Université de New York et École des sciences infirmières de l'Université de Nairobi, Kenya

Margaret Fitch, Collège des sciences infirmières Rory Meyers, Université de New York, New York, États-Unis et Faculté des sciences infirmières Bloomberg, Université de Toronto, Canada

Gaudence Niyonsenga, Hôpital King Faisal, Kigali, Rwanda

*Auteur-ressource : Pierre Céléstin Igiraneza, Camp de réfugiés Alight de Nyabiheke, Rwanda

Courriel : igiraneza7@gmail.com. Téléphone : +250788772820

DOI:10.5737/23688076313258265
Résultats : Parmi les répondantes, 55,5\% avaient une connaissance moyenne des facteurs de risque du cancer du sein, et 60,2\% sur l'incidence du dépistage $d u$ cancer du sein. On a constaté que $28 \%$ étaient familiarisées avec l'auto-examen des seins (AES) et $16,4 \%$ avec l'examen clinique des seins (ECS) et l'échographie mammaire. Au cours des deux années précédentes, les réponses entourant les pratiques de dépistage du sein ont révélé que $72 \%$ des répondantes n'avaient jamais réalisé d'AES et que 81,3\% n'avaient jamais passé d'ECS ou d'échographie mammaire. Pour 30,4\% des répondantes, l'information sur le dépistage du cancer du sein provenait principalement de la radio. Des relations significatives avec les pratiques de dépistage du cancer $d u$ sein sont ressorties pour l'âge et l'état matrimonial.

Conclusion : L'étude témoigne du peu de connaissances sur le cancer du sein, le dépistage et les pratiques relatives au dépistage de cette maladie. Il est donc nécessaire de promouvoir la sensibilisation au cancer du sein et de créer un programme qui puisse améliorer les connaissances et les pratiques en matière de dépistage afin d'améliorer la santé des femmes du sud de Kayonza.

Mots-clés : dépistage du cancer du sein, influence, pratiques.

\section{CONTEXTE}

A l'échelle mondiale, on estime que 627000 femmes sont Adécédées du cancer du sein en 2018, ce qui représente environ $15 \%$ de tous les décès attribuables au cancer chez les femmes (Bray et al., 2018). Pendant de nombreuses années, le cancer du sein était surtout considéré comme une maladie observée dans les pays développés, mais on rapporte maintenant un taux de mortalité plus élevé dans les pays en développement. Aux États-Unis, chez les femmes qui viennent de recevoir un diagnostic de cancer du sein, le taux de survie à 5 ans a grimpé à $89 \%$; ce taux avoisine plutôt les $40 \%$ dans les pays en développement (Miesfeldt et al., 2014). Chaque année, on estime à 1,7 million le nombre de nouveaux cas de cancer du sein diagnostiqués dans le monde. De tous les décès attribuables au cancer chez les femmes, 60 \% seraient des cancers du sein, majoritairement dans des pays en développement, où quelque 249260 nouveaux cas de cancer du sein y sont confirmés annuellement. La moitié de tous les cancers du sein et 62 $\%$ des décès causés par celui-ci sont enregistrés dans les pays en développement (Tfayli et al., 2010).

En Afrique, le cancer du sein demeure la plus importante cause de décès chez les femmes. Dans l'ensemble, on estime que des 882900 femmes habitant dans un pays en développement ayant reçu un diagnostic de cancer du sein, 324300 en sont décédées, majoritairement en Afrique de l'Est, du 
Nord et de l'Ouest (Donkor et al., 2015). Les taux approximatifs d'incidence normalisés selon l'âge par 100000 femmes s'élevaient à 30,4 en Afrique de l'Est, à 26 en Afrique centrale, à 38,6 en Afrique de l'Ouest et à 38,9 en Afrique du Sud (Donkor et al., 2015). En Afrique subsaharienne, on estime que 94000 femmes reçoivent un diagnostic de cancer du sein et que 48000 en meurent chaque année. En Afrique de l'Est, GLOBOCAN évalue le nombre de nouveaux cancers du sein à 19,9 \% chez les femmes de tous âges, alors que les taux d'incidence par 100000 personnes au Kenya et en Ouganda sont de $52 \%$ et $34 \%$ respectivement (Nambile et al., 2017). À titre d'exemple spécifique à un pays, les données de GLOBOCAN 2020 montrent qu'au Kenya, le taux d'incidence du cancer du sein avoisine les 40,3 par 100000 femmes, avec un taux de mortalité à 17,8 par 100000 personnes. Au Kenya, le cancer du sein représente environ $12 \%$ des nouveaux cas de cancer diagnostiqués annuellement, et environ 7,7 \% du nombre total de décès liés au cancer (Organisation mondiale de la santé, 2020).

$\mathrm{Au}$ Rwanda, les données de GLOBOCAN 2020 donnent un taux d'incidence du cancer du sein à 41,0 pour 100000 femmes, avec un taux de mortalité à 19,4 pour 100 000. L'incidence du cancer du sein au Rwanda représente 16,1\% de tous les nouveaux cas annuellement (Organisation mondiale de la santé, 2020). Une étude réalisée par le Centre d'excellence en soins du cancer de Butaro (Rwanda) révèle que des $82 \%$ des femmes ayant développé des tumeurs aux seins, $55 \%$ ont reçu un diagnostic de cancer du sein, et $36 \%$, un diagnostic de tumeur bénigne. Chez les femmes atteintes d'un cancer du sein, environ $20 \%$ en étaient au stade 1 ou 2 au moment du diagnostic, $46 \%$ avaient une maladie progressive localisée, et $31 \%$ avaient un cancer du sein métastatique (Pace, Dusengimana, Hategekimana et al., 2016).

En plus de réduire la morbidité et la mortalité attribuables au cancer du sein, la détection précoce des changements dans les seins et la rapidité de l'intervention améliorent le pronostic. L'American Cancer Society a fourni des stratégies pour la détection primaire par des pratiques de dépistage en fonction de l'âge des femmes (Majidi et al., 2017). Par contre, la compréhension des facteurs qui influencent les femmes du Rwanda à faire un dépistage du cancer du sein est limitée. L'objectif de cette étude consistait donc à évaluer les facteurs qui influencent les pratiques de dépistage du cancer du sein chez les femmes en âge de procréer du district sud de Kayonza.

\section{MATÉRIEL ET MÉTHODOLOGIE}

\section{Devis de l'étude}

Une étude transversale descriptive a été réalisée auprès de femmes âgées de 16 à 49 ans sélectionnées dans quatre centres de santé de la zone de recrutement de Rwinkwavu du district Sud de Kayonza, au Rwanda. La région desservie par l'hôpital de Rwinkwavu se trouve dans le même district, dans la province de l'est du Rwanda, peuplée de 211251 habitants. Léconomie de ce district repose principalement sur l'agriculture. On y cultive surtout la banane (29\%), le maïs (16\%), les haricots (24\%), le manioc (12\%) ainsi que les légumes (4\%), et le bétail principal est le bœuf. En plus des aliments locaux, la population, sur le plan géographique, a accès à des produits provenant d'autres régions du Rwanda qui, grâce au transport routier, sont offerts dans différents marchés et commerces (Institut National de la Statistique du Rwanda, 2012).

\section{Échantillon}

La taille de l'échantillon a été calculée à l'aide de la formule de Fisher (Fisher et al., 1998), où : Z (courbe de distribution normale) $=1,96$, pour un intervalle de confiance de $95 \%, P$ (estimation de la prévalence des mères dans la zone de recrutement de Rwinkwavu) $=20 \%$ et d (erreurs de fidélité) $=5 \%$. La taille de l'échantillon requis était de 246 femmes.

\section{Procédure de collecte de données}

La collecte de données s'est échelonnée sur deux mois. Dans chaque établissement de santé, le chercheur principal a choisi une aide-infirmière autorisée et l'a formée sur l'administration de l'outil de recherche. Les femmes qui se présentaient aux consultations externes des quatre centres de santé ont été invitées à participer à l'étude par le chercheur et son équipe. Une fois les renseignements sur l'étude divulgués, les participantes ont signé le formulaire de consentement. Elles ont ensuite été interrogées par l'auxiliaire qui s'occupait de faire remplir le questionnaire structuré, d'interroger les femmes et d'enregistrer leurs réponses. Le temps requis pour répondre au questionnaire était d'environ 10 minutes. L'auxiliaire remettait ensuite les questionnaires remplis et les formulaires de consentement éclairé signés au chercheur responsable.

\section{Formulaire de l'étude}

Dans le cadre de cette étude, l'instrument de collecte de données a été adapté par le chercheur, après avoir été examiné par une équipe d'experts de l'École de sciences infirmières et pratique sage-femme de l'Université du Rwanda. Il s'agit d'un outil ayant déjà été utilisé par des chercheurs du Nigéria et du Kenya (Paul, 2012) qui a été adapté au contexte du Rwanda. Le questionnaire détaillé utilisé pour cette étude comprenait cinq sections : section A - variables socioéconomiques; section B - connaissances sur les facteurs de risque du cancer du sein : quels facteurs influencent les pratiques de dépistage du cancer du sein et les méthodes de dépistage du cancer du sein; section C - sources d'information sur le cancer du sein; section D - influence des établissements de santé sur les pratiques de dépistage du cancer du sein; et section $\mathrm{E}$ - facteurs socioéconomiques et culturels qui empêchent les femmes de procéder au dépistage du cancer du sein.

On a mené une évaluation de la validité apparente et du contenu dans quatre centres de santé lors d'un essai pilote mené auprès de vingt-cinq répondantes venues consulter des spécialistes en soins infirmiers oncologiques. Cette évaluation était nécessaire pour confirmer que le questionnaire de recherche mesurait bien ce qu'il devait mesurer; elle consistait à réviser chaque question et à vérifier si l'instrument de collecte de données était approprié ou non. Les questionnaires à l'essai ont été distribués, puis les participantes ont été invitées à donner leur avis sur l'orientation et la précision des questions. Leurs commentaires ont servi à améliorer le questionnaire. Les données obtenues par l'intermédiaire de 
l'essai pilote ont été analysées au moyen de la version 21.0 du Statistical Package for Social Sciences (SPSS); le coefficient de fiabilité était de 0,8 .

\section{Analyse des données}

Les données des participantes à l'étude ont été transformées en information significative, pour faciliter l'interprétation et la compréhension. Par la suite, les données ont été groupées, organisées, ordonnées et enregistrées sur une feuille prévue à cet effet. Une analyse basée sur les statistiques descriptives et inférentielles a été réalisée à l'aide du programme informatique SPSS (version 21.0); les résultats ont été présentés sous forme de graphiques et de tableaux. On a calculé les fréquences pour toutes les réponses données aux questions, ainsi que les scores d'évaluation des connaissances en ajoutant les scores correspondant aux items évalués. Un score moyen de connaissances supérieur à $80 \%$ était considéré comme un « bon » niveau de connaissances, un score entre $50 \%$ et $80 \%$, un niveau «moyen », et les scores inférieurs à $50 \%$, un niveau «faible».

\section{Considérations éthiques}

Le Comité d'évaluation de la recherche (CÉR) du Collège de médecine et de sciences de la santé de l'Université du Rwanda a autorisé cette recherche. La collecte des données a été approuvée par le directeur général de l'hôpital de district de Rwinkwavu. Les objectifs de l'étude ont été expliqués à chacune des participantes, qui ont aussi été avisées de leur droit de se retirer de l'étude et assurées de la confidentialité et de l'anonymat relativement aux renseignements fournis. Avant la collecte de données, les femmes ont signé le formulaire de consentement éclairé à l'étude.

\section{RÉSULTATS}

\section{Caractéristiques démographiques}

Les répondantes ont été divisées dans des groupes d'âge de 16 à 49 ans (tableau 1). Le tiers d'entre elles (32,9 \%) faisaient partie du groupe des 30-39 ans. Le plus petit pourcentage $(7,7 \%)$ était constitué des $40-49$ ans. Alors que $61,8 \%$ des femmes interrogées n'avaient fréquenté que l'école primaire, 14,2 \% n'avaient pas reçu cette instruction de base. Parmi le groupe, $24 \%$ des répondantes avaient fait des études secondaires ou universitaires. Environ la moitié $(56,1 \%)$ des femmes sondées se situaient dans la catégorie trois du programme Ubudehe (catégorisation de la population en fonction de la pratique et de la culture de l'action collective et du soutien mutuel pour résoudre les problèmes au sein de la collectivité). Le pourcentage de femmes appartenant aux catégories 2 et 1 atteignait $33,3 \%$ et $10 \%$ respectivement. Par ailleurs, $77,6 \%$ bénéficiaient d'une assurance maladie communautaire.

Létude a révélé que 34,1\% des participantes à l'étude ne faisaient pas confiance au dépistage du cancer du sein. Bien que 32,6\% des répondantes aient déjà passé un ECS et une échographie mammaire, $81,3 \%$ des femmes n'étaient pas familières avec ces méthodes. Au cours des deux années précédentes, 200 répondantes $(81,3 \%$ du groupe) n'avaient jamais fait l'AES, alors que 14,6\% d'entre elles avaient fait l'AES chaque année (tableau 1).

\begin{tabular}{|c|c|c|}
\hline \multicolumn{3}{|c|}{$\begin{array}{l}\text { Tableau } 1 \text { : Données sociodémographiques et pratiques de } \\
\text { dépistage du cancer du sein chez les répondantes }(n=246)\end{array}$} \\
\hline Catégories démographiques & Fréquence & Pourcentage (\%) \\
\hline \multicolumn{3}{|l|}{ Âge } \\
\hline $16-19$ & 70 & 28,5 \\
\hline $20-29$ & 76 & 30,9 \\
\hline $30-39$ & 81 & 32,9 \\
\hline $40-49$ & 19 & 7,7 \\
\hline \multicolumn{3}{|l|}{ Niveau d'éducation } \\
\hline Aucune éducation de base & 35 & 14,2 \\
\hline Primaire & 152 & 61,8 \\
\hline Secondaire & 50 & 20,3 \\
\hline Universitaire & 9 & 3,7 \\
\hline \multicolumn{3}{|l|}{ État matrimonial } \\
\hline Célibataire & 58 & 23,6 \\
\hline Mariée & 153 & 62,2 \\
\hline Séparée & 18 & 7,3 \\
\hline Veuve & 17 & 6,9 \\
\hline \multicolumn{3}{|l|}{ Ubudehe (catégories sociales) } \\
\hline Catégorie 1 & 26 & 10,6 \\
\hline Catégorie 2 & 82 & 33,3 \\
\hline Catégorie 3 & 138 & 56,1 \\
\hline Catégorie 4 & 0 & 0 \\
\hline \multicolumn{3}{|l|}{ Assurance maladie } \\
\hline Autres & 7 & 2,8 \\
\hline $\mathrm{CHBI}$ & 191 & 77,6 \\
\hline RAMA & 28 & 11,4 \\
\hline $\mathrm{MMI}$ & 8 & 3,3 \\
\hline UAP & 3 & 1,2 \\
\hline AUCUNE & 9 & 3,7 \\
\hline \multicolumn{3}{|l|}{$\begin{array}{l}\text { Pratique le dépistage du cancer } \\
\text { du sein (par groupe d'âge) }\end{array}$} \\
\hline $16-19$ & 20 & 28 \\
\hline $20-29$ & 76 & 30,9 \\
\hline $30-39$ & 81 & 32,6 \\
\hline $40-49$ & 19 & 7,7 \\
\hline \multicolumn{3}{|l|}{ Confiance envers le dépistage } \\
\hline Oui & 43 & 17,5 \\
\hline Non & 84 & 34,1 \\
\hline \multicolumn{3}{|l|}{$\begin{array}{l}\text { Déjà passé un ECS/une } \\
\text { échographie mammaire }\end{array}$} \\
\hline Au moins une fois & 36 & 14,6 \\
\hline Jamais & 200 & 81,3 \\
\hline Pas certaine & 10 & 4,1 \\
\hline
\end{tabular}


Connaissance des risques de cancer du sein

Environ la moitié $(56,1 \%)$ des répondantes interrogées ( $\mathrm{N}=246)$ connaissaient les facteurs de risque du cancer $\mathrm{du}$ sein (tableau 2), alors que 43,9\% des femmes les ignoraient. Aucune association statistiquement significative n'a pu être faite entre les connaissances et les variables démographiques.

Le tableau 3 présente les facteurs qui influencent les pratiques de dépistage du cancer du sein. Près du trois quarts (74,4 \%) des répondantes s'accordaient pour dire qu'un écoulement anormal les inciterait à passer un ECS ou une échographie. Pour 70,7 \% d'entre elles, une masse ou une douleur au sein serait aussi considérée comme un incitateur. Seulement 39,9\% des participantes se disaient influencées par l'échec de méthodes de guérison traditionnelles pour les tumeurs du sein et le fait que ces interventions n'avaient pas l'effet escompté.

La plupart des répondantes (60,2 \%) n'étaient pas très au fait de l'impact du dépistage du cancer du sein, alors que 55,5\% des femmes connaissaient moyennement les facteurs de risque de la maladie. Les connaissances sur l'AES (20,8\%) et l'échographie (16,5\%) étaient faibles (figure 4.1).
Pratiques de dépistage du cancer du sein chez les répondantes

Environ un tiers des répondantes dans chaque groupe d'âge de 16 et 39 ans et 7,7\% des femmes de 40 à 49 ans ont bénéficié d'un dépistage du cancer du sein (tableau 1). Un quart (25,6\%) des répondantes se sentaient à l'aise de pratiquer l'AES et 14,6 \% avaient déjà eu un ECS ou une échographie mammaire. La plupart des répondantes (79\%) ne prévoyaient ni passer d'ECS ni d'échographie, alors que $8 \%$ envisageaient l'examen clinique des seins. Seulement $3 \%$ des participantes hésitaient à passer un ECS ou une échographie mammaire (figure 4.2).

Influence de l'éducation sur le dépistage du cancer du sein dans les établissements de santé

Le tableau 4 montre que les répondantes qui ont reçu de l'information sur la santé du sein dans des cliniques de maladies non transmissibles ou de maternité sont plus susceptibles d'adhérer aux pratiques de dépistage de cancer du sein, ce qui démontre une association statistiquement significative avec ces pratiques $(=34,748 ; p=.001)$. Deux tiers $(62,6 \%)$ des femmes qui ne pratiquaient pas l'AES n'avaient jamais été renseignées sur la santé du sein dans un établissement de santé.

Tableau 2 : Connaissance des facteurs de risque du cancer du sein

\begin{tabular}{|l|c|c|c|c|c|}
\hline $\begin{array}{l}\text { Connaissance des facteurs } \\
\text { de risque du cancer du sein }\end{array}$ & $\begin{array}{c}\text { Entièrement en désaccord } \\
\mathbf{n}(\%)\end{array}$ & $\begin{array}{c}\text { En désaccord } \\
\mathbf{n}(\%)\end{array}$ & $\begin{array}{c}\text { Neutre } \\
\mathbf{n}(\%)\end{array}$ & $\begin{array}{c}\text { En accord } \\
\mathbf{n}(\%)\end{array}$ & $\begin{array}{c}\text { Totalement en accord } \\
\mathbf{n}(\%)\end{array}$ \\
\hline 1. Inactivité physique & $30(12,2)$ & $46(18,7)$ & $52(21,1)$ & $106(43,1)$ & $12(4,9)$ \\
\hline 2. Consommation d'alcool & $8(3,3)$ & $34(13,8)$ & $35(14,2)$ & $133(54,1)$ & $36(14,6)$ \\
\hline 3. Allaitement & $9(3,7)$ & $57(23,2)$ & $31(12,6)$ & $112(45,5)$ & $37(15,0)$ \\
\hline 4. Malédiction & $8,1(20,0)$ & $66(26,8)$ & $45(18,3)$ & $85(34,6)$ & $30(12,2)$ \\
\hline 5. Tabagisme & $13(5,3)$ & $46(18,7)$ & $21(8,5)$ & $119(48,4)$ & $47(19,1)$ \\
\hline 6. Hérédité & $23(9,3)$ & $57(23,2)$ & $38(15,4)$ & $98(39,8)$ & $30(12,2)$ \\
\hline 7. Blessure à la poitrine & $17(6,9)$ & $50(20,3)$ & $30(12,2)$ & $109(44,3)$ & $40(16,3)$ \\
\hline 8. Menstruations précoces & $30(12,2)$ & $77(31,3)$ & $29(11,8)$ & $77(31,3)$ & $33(13,4)$ \\
\hline Total & 7,6 & 22,0 & 14,3 & 42,6 & 13,5 \\
\hline
\end{tabular}

Tableau 3. Connaissances des répondantes sur l'impact des pratiques de dépistage du cancer du sein

\begin{tabular}{|l|c|c|c|c|c|}
\hline VARIABLES & & & \\
\hline $\begin{array}{l}\text { Connaissances de l'impact du } \\
\text { dépistage du cancer du sein }\end{array}$ & $\begin{array}{c}\text { Entièrement en désaccord } \\
\mathbf{n}(\%)\end{array}$ & $\begin{array}{c}\text { En désaccord } \\
\mathbf{n}(\%)\end{array}$ & $\begin{array}{c}\text { Neutre } \\
\mathbf{n}(\%)\end{array}$ & $\begin{array}{c}\text { En accord } \\
\mathbf{n}(\%)\end{array}$ & $\begin{array}{c}\text { Totalement en accord } \\
\mathbf{n}(\%)\end{array}$ \\
\hline 1. Douleur à un sein & $20(8,1)$ & $43(17,5)$ & $9(3,7)$ & $136(55,3)$ & $38(15,4)$ \\
\hline 2. Antécédents familiaux & $39(15,9)$ & $74(30,1)$ & $14(5,7)$ & $84(34,1)$ & $35(14,2)$ \\
\hline 3. Masse dans un sein & $20(8,1)$ & $45(18,3)$ & $7(2,8)$ & $130(52,8)$ & $44(17,9)$ \\
\hline 4. Écoulements anormaux & $19(7,7)$ & $35(14,2)$ & $9(3,7)$ & $136(55,3)$ & $47(19,1)$ \\
\hline 5. Importance et valeur & $24(9,8)$ & $62(25,2)$ & $14(5,7)$ & $96(39,0)$ & $50(20,3)$ \\
\hline 6. Échec de la phytothérapie & $30(12,2)$ & $103(41,9)$ & $15(6,1)$ & $73(29,7)$ & $25(10,2)$ \\
\hline Total & 10,3 & 24,5 & 4,6 & 44,4 & 16,2 \\
\hline
\end{tabular}




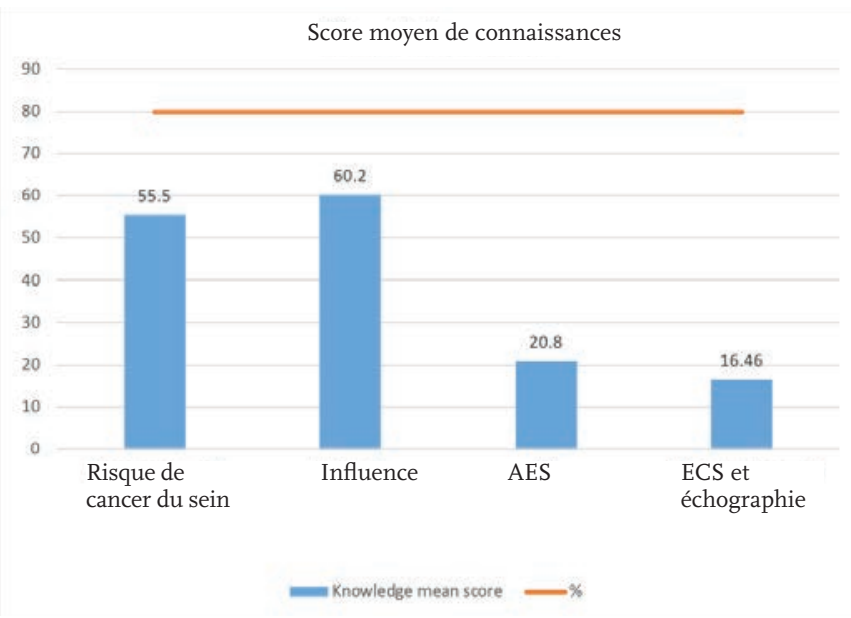

Figure 4.1. Scores de connaissances

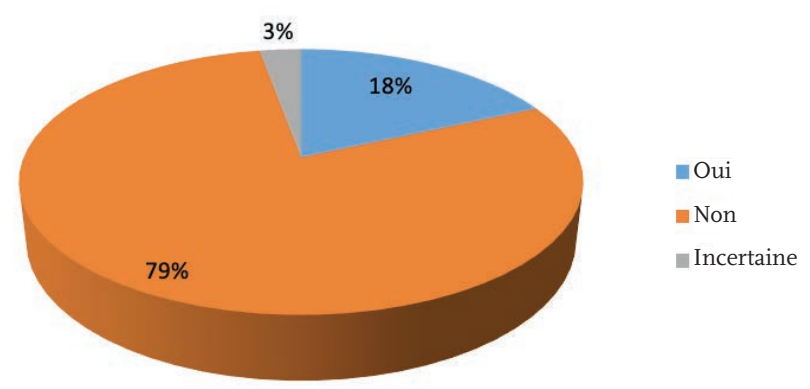

Figure 4.2 Intention de passer un examen clinique des seins ou une échographie mammaire

\begin{tabular}{|c|c|c|c|}
\hline \multicolumn{4}{|c|}{$\begin{array}{l}\text { Tableau } 4 \text { : Lien entre l'enseignement reçu dans les } \\
\text { établissements de santé et l'examen de dépistage mammaire }\end{array}$} \\
\hline \multirow[t]{2}{*}{ VARIABLE } & \multicolumn{3}{|c|}{ AUTO-EXAMEN DES SEINS } \\
\hline & $\begin{array}{l}n(\%) \\
\text { ddl }\end{array}$ & Total (\%) & valeur $p$ \\
\hline $\begin{array}{l}\text { Éducation à la santé des } \\
\text { seins dans une clinique } \\
\text { de MNT ou une clinique } \\
\text { de maternité }\end{array}$ & & & \\
\hline Offert & $\begin{array}{c}92(37,4 \%) \\
34,748 \text { a }\end{array}$ & $\begin{array}{l}246(100 \%) \\
12\end{array}$ & .001 \\
\hline Non offert & $154(62,6 \%)$ & & \\
\hline $\begin{array}{l}\text { Fourniture d'aides } \\
\text { visuelles dans une } \\
\text { clinique de MNT ou une } \\
\text { clinique de maternité }\end{array}$ & & & \\
\hline Offert & $\begin{array}{l}90(36,6) \\
45,597^{a}\end{array}$ & $\begin{array}{l}246(100 \%) \\
12\end{array}$ & .000 \\
\hline Non offert & $156(63.4 \%)$ & & \\
\hline $\begin{array}{l}\text { Informations sur le } \\
\text { dépistage du cancer } \\
\text { du sein de la part } \\
\text { des médecins et des } \\
\text { infirmières }\end{array}$ & & & \\
\hline Offert & $\begin{array}{c}48(19,5 \%) \\
6.521^{\mathrm{a}}\end{array}$ & $\begin{array}{l}246(100 \%) \\
12\end{array}$ & .008 \\
\hline Non offert & $198(80,5 \%)$ & & \\
\hline
\end{tabular}

\begin{tabular}{|c|c|c|c|c|c|}
\hline VARIABLES & $\begin{array}{l}\text { Entièrement en désaccord } \\
n(\%)\end{array}$ & $\begin{array}{c}\text { En désaccord } \\
n(\%)\end{array}$ & $\begin{array}{l}\text { Neutre } \\
n(\%)\end{array}$ & $\begin{array}{c}\text { En accord } \\
n(\%)\end{array}$ & $\begin{array}{c}\text { Totalement en accord } \\
\text { n (\%) }\end{array}$ \\
\hline Pratique cultuelle & $58(23,6)$ & $133(54,1)$ & $26(10,6)$ & $28(11,4)$ & $1(0,4)$ \\
\hline Manque de connaissances & $16(6,5)$ & $96(39,0)$ & $13(5,3)$ & $105(42,7)$ & $16(6,5)$ \\
\hline Longue distance & $15(6,1)$ & $93(37,0)$ & $20(8,1)$ & $93(37,8)$ & $27(11,0)$ \\
\hline Manque de cliniques du sein & $11(4,5)$ & $42(17,1)$ & $16(6,5)$ & $138(56,1)$ & $39(15,9)$ \\
\hline L'échographie est douloureuse & $44(17,9)$ & $120(48,8)$ & $37(15,0)$ & $34(13,8)$ & $11(4,5)$ \\
\hline $\begin{array}{l}\text { Longs délais d'attente dans les } \\
\text { établissements de santé }\end{array}$ & $25(10,2)$ & $78(31,7)$ & $17(6,9)$ & $97(39,4)$ & $29(11,8)$ \\
\hline
\end{tabular}


Environ 63,4 \% des femmes qui n'avaient jamais fait d'AES n'avaient jamais vu comment se déroulait le dépistage du cancer du sein $=45,597, p=.000$ ). On remarque une association statistiquement significative entre le fait d'être conseillée sur le dépistage du cancer du sein et les pratiques d'AES $=45,597$, $p=.008)$. Enfin, $80 \%$ des répondantes n'avaient pas été renseignées sur l'auto-examen des seins.

Chez $72 \%$ des participantes, le manque de cliniques du sein était considéré comme le principal obstacle au dépistage (tableau 5). Le manque de connaissances sur le dépistage était également considéré comme un obstacle pour 49,2\% d'entre elles, de même que la grande distance à parcourir pour accéder aux établissements de santé (48,8\%). Aucune signification statistique entre le statut socioéconomique et la méthode utilisée pour dépister le cancer du sein n'a été démontrée.

Parmi les répondantes renseignées sur la santé du sein et le dépistage du cancer du sein, 30,4\% avaient été informées par la radio. Au total, 35,4\% des participantes n'avaient été exposées à aucune source d'information sur le cancer du sein et le dépistage de cette maladie. Les autres sources d'information étaient la télévision (15\%), le journal (11,8\%), les amis $(0,8 \%)$ et le milieu hospitalier $(1,2 \%)$.

\section{DISCUSSION}

Les résultats indiquent que les facteurs sociodémographiques comme les catégories sociales (Ubudehe), l'assurance maladie, le niveau d'éducation, l'âge et l'état matrimonial influencent fortement la pratique de l'auto-examen des seins. En effet, la relation entre l'âge et l'auto-examen des seins s'est révélée statistiquement significative. Les femmes de 20-29 ans sont celles qui pratiquaient le plus l'auto-examen des seins, tandis que les femmes de 40-49 ans étaient les moins assidues. Le fait d'être mariée aurait un impact positif sur l'auto-examen et l'échographie. De plus, le dépistage du cancer du sein serait plus fréquent chez les femmes mariées que chez les célibataires, peut-être à cause d'une plus grande conscience de leur responsabilité de rester en bonne santé et de vivre longtemps pour subvenir aux besoins de leurs enfants et de la famille.

La présente étude a révélé que 55,5 \% des répondantes ont une connaissance moyenne des facteurs de risque du cancer du sein. Cette conclusion s'apparente à celle d'une étude nigérienne sur le niveau de connaissances des pratiques de dépistage du cancer du sein, qui s'est avéré faible pour $84 \%$ des femmes, moyennes pour seulement $23 \%$ et bonnes pour 0,7 \% d'entre elles (Kohler et al., 2017a). Le petit écart pourrait être lié aux caractéristiques de l'échantillon dans les deux études et à un degré de sensibilisation différent face à ce cancer.

Parmi toutes les participantes, 68,7 \% considèrent la consommation d'alcool comme un facteur de risque majeur, suivi du tabagisme (67,5\%). Nos résultats diffèrent de ceux d'une étude éthiopienne en ce qui concerne la connaissance des facteurs de risque. En effet, 71,3\% des femmes sondées ont nommé le tabagisme comme principal facteur de risque alors que 44,2 \% d'entre elles ont répondu que la consommation d'alcool était un facteur de risque majeur (Tabrizi, 2015). Cela pourrait s'expliquer par les différences entre les facteurs sociodémographiques et les domaines d'étude.

Des répondantes ayant été informées sur l'auto-examen des seins, $81 \%$ ont confirmé n'avoir jamais pensé à cette méthode de dépistage. La présente étude a dévoilé une corrélation significative entre le niveau d'éducation et le fait d'avoir été renseignée sur l'auto-examen des seins. Ainsi, $72 \%$ des répondantes ne l'avaient jamais pratiqué $\left(\chi^{2}=22,457, \mathrm{p}=.000\right), 17,5 \%$ faisaient l'AES une fois par année, 6,1 \% le faisaient mensuellement et 4,1 \%, sur une base hebdomadaire. Plus des trois quarts $(84,3 \%)$ d'entre elles ne se sentaient pas confiantes face à l'AES. Nos résultats sont supérieurs à ceux d'une étude de l'Université de Buea où seulement $9 \%$ des participantes savaient comment faire l'AES et que $3 \%$ le pratiquaient régulièrement (Angahar, 2017). Lécart pourrait être dû aux différents niveaux de sensibilisation au cancer du sein entre les deux populations à l'étude.

La majorité des répondantes $(81,3 \%)$ de notre étude n'utilisaient ni l'auto-examen des seins ni l'échographie mammaire comme moyen de dépistage. De plus, $79 \%$ des participantes ne prévoyaient pas passer d'ECS ni d'échographie mammaire dans les deux prochaines années. Notre conclusion correspond donc à celle d'une étude menée au Kenya, où $72 \%$ des femmes rapportaient n'avoir jamais subi d'examen clinique des seins (Tabrizi, 2015). Cela pourrait être attribuable aux caractéristiques similaires des échantillons comme le faible niveau d'éducation, la pauvreté et la défaillance des installations où l'on réalise les tests diagnostiques. Les deux études ont été menées dans des régions rurales où la population était peu sensibilisée au dépistage du cancer du sein, comparativement à celle des villes.

Parmi les répondantes, 74,4\% affirmaient qu'un écoulement anormal les convaincrait de passer un ECS ou une échographie mammaire, alors que 70,7 \% d'entre elles seraient portées à le faire si elles sentaient une masse ou une douleur au sein. Nos résultats vont dans le même sens que ceux d'Ibrahim et collaborateurs (2013), qui concluent que les femmes sont plus enclines au dépistage lorsqu'une bosse est détectée. Cela concorde également avec une étude nigérienne où l'écoulement sanguin du mamelon (21,1\%), les symptômes liés à une bosse $(40,1 \%)$ et des douleurs au sein (57\%) sont ressortis comme des facteurs incitant au dépistage (Kohler et al., 2017b).

Les résultats de l'étude indiquent que les répondantes qui étaient renseignées sur la santé du sein, le cancer du sein et le dépistage de la maladie ont eu différentes sources d'information, surtout la radio. Ces constats s'apparentent à ceux d'une étude réalisée au Ghana, où 39,8 \% des participantes considéraient la radio comme la principale source d'information sur le cancer du sein; les autres sources nommées étaient la télévision (17,5\%), la famille et les amis (16,7\%), les journaux et les magazines (14,2\%). Seulement $5 \%$ d'entre elles avaient été renseignées par les médecins et les infirmières (Bray et al., 2018). Les raisons derrière ces faibles résultats pourraient être reliées à l'indisponibilité et à l'inaccessibilité des publicités sur le dépistage du cancer du sein à la radio, à la télévision et dans les journaux. On pense aussi que les travailleurs de la santé ne font pas assez d'enseignement sur les méthodes de dépistage de ce cancer. 
Une étude menée en Égypte a révélé que les femmes qui ont peu de connaissances en santé et sur les grandes lignes du dépistage du cancer du sein auraient du mal à accéder aux établissements de santé et à comprendre les compétences liées au cancer du sein (Bray et al., 2018). Une étude réalisée en Afrique subsaharienne précise que les facteurs associés au manque de connaissances et d'établissements de santé influencent la prise en charge du cancer du sein (Angahar, 2017). Nos résultats rejoignent ces conclusions; en effet, l'éducation en matière de santé du sein prodiguée dans les cliniques de maladies non transmissibles (MNT) et les cliniques de maternité amène les femmes à pratiquer davantage l'AES $(\chi 2=34,748, p=.001)$. De plus, $62,6 \%$ des femmes qui ne pratiquaient pas l'auto-examen des seins n'avaient jamais reçu d'enseignement sur la santé des seins dans un établissement de soins de santé. Nous observons une corrélation négative significative avec l'AES $(\chi 2=45,59$, $p=.000$ ). Environ $63,4 \%$ des femmes qui ne pratiquaient pas l'AES n'avaient jamais reçu d'aide visuelle sur le dépistage. On sait pourtant que le fait d'être renseigné sur le sujet a un impact positif important sur les pratiques de dépistage de ce cancer. Malheureusement, $80 \%$ des répondantes n'avaient pas été informées sur l'auto-examen des seins $(\chi 2=45,597, p=.008)$. Des séances d'informations sur la santé du sein devront être offertes sur une base régulière dans les services de maternité et les cliniques de MNT afin de sensibiliser la population et d'améliorer les pratiques de dépistage du cancer du sein. Dans la présente étude, la majorité des répondantes neétaient pas renseignées sur la santé du sein, leur rôle dans la prévention et le contrôle de ce cancer ainsi que sur la façon d'intégrer l'AES dans leur routine quotidienne.

L'American Cancer Society souligne que les croyances culturelles et religieuses, les besoins complexes en matière de santé et les carences socioéconomiques sont reliés aux faibles niveaux de connaissances sur le cancer du sein et de compétences en matière de recherche sur la santé (Cumber et al., 2017). Ces résultats contrastent avec ceux de notre étude, où le manque de cliniques de dépistage de cancer du sein est considéré comme le principal obstacle pour la majorité des femmes (72\%). Les participantes ont aussi mentionné que le manque de connaissances sur le dépistage du cancer du sein $(49,2 \%)$

\section{RÉFÉRENCES}

Angahar, L. T. (2017). An overview of breast cancer epidemiology, risk factors, pathophysiology, and cancer risks reduction. MOJ Biology and Medicine, 1(4).

Bray, F., Ferlay, J., Soerjomataram, I., Siegel, R. L., Torre, L. A., \& Jemal, A. (2018). Global cancer statistics 2018: GLOBOCAN estimates of incidence and mortality worldwide for 36 cancers in 185 countries. CA: A Cancer Journal for Clinicians, 68(6), 394-424. https://doi.org/10.3322/caac.21492

Country-specific, I., Method, R., \& Total, M. (2020). 12952 209. 237, $1-2$.

Cumber, S. N., Nchanji, K. N., \& Tsoka-Gwegweni, J. M. (2017). Breast cancer among women in sub-Saharan Africa: Prevalence and a situational analysis. Southern African Journal of Gynaecological Oncology, 9(2), 35-37. https://doi.org/10.1080/20742835.2017.13914 67 et les grandes distances qui les séparent des établissements de santé $(48,8 \%)$ étaient des facteurs dissuasifs importants au dépistage. Shamseddine et collaborateurs (2010) ont mentionné que les facteurs qui entravent le dépistage du cancer du sein s'expliquent entre autres par le manque de sensibilisation à la maladie, une faible littératie en santé et en éducation, des lacunes dans les programmes de diagnostic, l'absence de soutien gouvernemental, le manque de financement et les obstacles sociaux au dépistage précoce découlant d'une faible priorité accordée à la santé des femmes. Ces constats reflètent la réalité des pays à faibles revenus. Ils correspondent à nos résultats de recherche indiquant que plusieurs femmes ne connaissent pas les pratiques de dépistage du cancer du sein et que le dépistage n'est peut-être pas offert dans leurs centres de santé.

\section{RECOMMANDATIONS}

Il est nécessaire de créer et d'intensifier les campagnes de sensibilisation au cancer du sein dans les différentes plateformes médiatiques disponibles, en écrivant par exemple de courts messages sur les médias sociaux, ou par l'entremise de campagnes de sensibilisation dans les établissements de santé et les écoles. Cela améliorera non seulement les pratiques de dépistage du cancer du sein, mais aussi les connaissances sur les manifestations, les facteurs de risque, les avantages du dépistage précoce et le traitement de la maladie, pour des résultats plus réjouissants.

\section{CONCLUSION}

Létude a révélé un manque de connaissances des répondantes à propos des pratiques de dépistage du cancer du sein. Plus particulièrement, les faibles connaissances sur les facteurs de risque de la maladie influencent ces pratiques de dépistage et les tests associés. Beaucoup de femmes ne consultent aucune source d'informations sur le cancer du sein et les méthodes de dépistage. Parmi celles qui étaient renseignées sur ce cancer, les informations avaient surtout été entendues à la radio. Seule une faible proportion de femmes pratiquait occasionnellement l'auto-examen des seins.

Donkor, A., Wiafe, S., Yarney, J., Opoku, Y., Antwi, W., \& Kyei, K. A. (2015). iMedPub journals factors contributing to late presentation of breast cancer in Africa: A systematic literature review. Abstract. 1-10.

Feigin, V. L., Forouzanfar, M. H., Krishnamurthi, R., Mensah, G. A., Connor, M., Bennett, D. A., \& Murray, C. (2014). Global and regional burden of stroke during 1990-2010: Findings from the Global Burden of Disease Study 2010. The Lancet, 383(9913), 245-255.

Kohler, R. E., Gopal, S., Lee, C. N., Weiner, B. J., Reeve, B. B., \& Wheeler, S. B. (2017a). Breast cancer knowledge, behaviors, and preferences in Malawi: Implications for early detection interventions from a discrete choice experiment. Journal of Global Oncology, 3(5), 480-489. https://doi.org/10.1200/JGO.2016.005371

Kohler, R. E., Gopal, S., Lee, C. N., Weiner, B. J., Reeve, B. B., \& Wheeler, S. B. (2017b). Breast cancer knowledge, behaviors, 
and preferences in Malawi: Implications for early detection interventions from a discrete choice experiment. Journal of Global Oncology, 3(5), 480-489. https://doi.org/10.1200/igo.2016.005371

Majidi, A., Majidi, S., Salimzadeh, S., Pool, M. K.-, Sadjadi, A., Salimzadeh, H., \& Delavari, A. (2017). Cancer screening awareness and practice in a middle income country: A systematic review from Iran. Asian Pac J Cancer Prev, 18, 3187-3194. https://doi. org/10.22034/APJCP.2017.18.12.3187

Miesfeldt, S., Morse, E., Maegga, B., Joseph, , Gertrud, \& Miesfeldt, S. (2014). Breast cancer knowledge, beliefs, and screening practices among women seeking care at district hospitals in Dar es Salaam, Tanzania. Breast Cancer: Basic and Clinical Research, 73. https://doi. org/10.4137/BCBCR.S13745

Nambile, S., Nkeh, K., Tsoka-gwegweni, J. M., \& Africa, S. (2017). Breast cancer among women in sub-Saharan Africa: Prevalence and a situational analysis. Southern African Journal of
Gynaecological Oncology, 9(2), 35-37. https://doi.org/10.1080/20742 835.2017.1391467

Pace, L. E., Dusengimana, J. M. V., Hategekimana, V., Habineza, H., Bigirimana, J. B., Tapela, N., ... \& Mpunga, T. (2016). Benign and malignant breast disease at Rwanda's first public cancer referral center. The oncologist, 21(5), 571.

Shamseddine, A., Tfayli, A., Temraz, S., \& Abou Mrad, R. (2010). Breast cancer in low- and middle-income countries: An emerging and challenging epidemic. Journal of Oncology, 2010. https://doi. org/10.1155/2010/490631

Tfayli, A., Temraz, S., Mrad, R. A., \& Shamseddine, A. (2010). Breast cancer in low- and middle-income countries: An emerging and challenging epidemic. Journal of Oncology, 2010. https://doi. org/10.1155/2010/490631

World Health Organization. (2020). WHO report on cancer: Setting priorities, investing wisely and providing care for all. 